\title{
Exposición a contaminantes provenientes de termoeléctricas a carbón y salud infantil: ¿Cuál es la evidencia internacional y nacional?
}

\author{
Exposure to contaminants from coal-fired thermoelectric plants and children's health: \\ What is the international and national evidence?
}

\author{
Sandra Cortés A. ${ }^{\mathrm{a}, \mathrm{b}, \mathrm{eff}}$, Karla Yohannessen V. ${ }^{\mathrm{a}, \mathrm{c}, \mathrm{d}}$, Lidya Tellerías C. ${ }^{\mathrm{a}}$, Ericka Ahumada P.,b \\ aComité Salud Ambiental Infantil, Sociedad Chilena de Pediatría. \\ bFacultad de Medicina. Pontificia Universidad Católica de Chile. \\ cPrograma de Salud Ambiental, Escuela de Salud Pública, Facultad de Medicina, Universidad de Chile. \\ dDepartamento de Pediatría y Cirugía Infantil, Facultad de Medicina, Universidad de Chile \\ eAdvanced Center for Chronic Diseases (ACCDIS). \\ ${ }^{\mathrm{f} C}$ entro de Desarrollo Sostenible (CEDEUS).
}

Recibido: 23 de mayo de 2018; Aceptado: 19 de noviembre de 2018

\begin{abstract}
Resumen
Las centrales termoeléctricas (CTE) a carbón representan un riesgo para la salud de las comunidades expuestas. Se realizó una revisión de la literatura científica nacional e internacional enfocada en los efectos en salud de niños y la exposición a emisiones al aire provenientes de CTE a carbón. Se incluyeron 21 artículos para su revisión en texto completo, donde se midieron efectos en salud infantil relacionados a presencia de biomarcadores de exposición y efecto, daños perinatales, neuroconductuales y respiratorios principalmente. La exposición a emisiones de CTE a carbón en el embarazo se asoció a niños con bajo peso y muy bajo peso al nacer, menor talla, menor diámetro de Circunferencia del Cráneo (CC) y prematuridad; el diámetro de CC aumentó en recién nacidos después del cierre de CTE. Se encontraron menor coeficiente de desarrollo (CD) y coeficiente intelectual (CI) en niños expuestos a emisiones de CTE a carbón comparados con no expuestos; CD aumentó cuando la central fue cerrada. Por otro lado, vivir en zonas con fuentes de emisión de mercurio (asociadas a CTE y plantas de cemento que funcionan con carbón) se asoció con mayor riesgo de autismo. En salud respiratoria, los artículos fueron consistentes en reportar menor función pulmonar en niños residentes en zonas expuestas a fuentes de combustión de carbón comparados con grupos de niños no expuestos. Es muy necesario abrir el debate en Chile sobre los riesgos controlables a los que se enfrenta la población infantil a consecuencia de plantas generadoras de energía instaladas en Chile.
\end{abstract}

Palabras clave: Termoeléctricas; Carbón; Salud Infantil; Exposición 


\begin{abstract}
Coal-fired power plants (CFPP) represent a health risk to the exposed communities. A review of national and international scientific literature was made focused on the health effects on children and exposure to air emissions from CFPP. Twenty-one articles were included for full-text review, where effects on child health mainly related to the biomarkers presence of exposure and effect, perinatal, neurobehavioral and respiratory damages were measured. Exposure to CFPP emissions in pregnancy was associated with low birth weight and very low birth weight, shorter height, smaller head circumference (HC) diameter, and prematurity; the HC diameter increased in newborns after the CFPP closure. Lower coefficient of development (CD) and intelligence quotient (IQ) were found in children exposed to CFPP emissions compared with unexposed ones; CD increased when the plant was closed. On the other hand, living in areas with mercury emission sources (associated with CFPP and cement plants that work with coal) was associated with an increased risk of autism. In respiratory health, the articles were consistent with reporting lower pulmonary function in children living in areas exposed to coal combustion sources compared with groups of unexposed children. There is a great need to open the debate in Chile on the controllable risks faced by the child population as a result of power generation plants located in Chile.
\end{abstract}

Keywords:

Thermoelectric Power

Plants;

Coal;

Child Health;

Exposure

\section{Introducción}

Una central termoeléctrica (CTE) es una instalación empleada en la generación de electricidad a partir de la energía liberada en forma de calor, mediante la combustión de combustibles fósiles como petróleo, gas natural o carbón. Las CTE, basadas en el uso de carbón representan un riesgo a la salud en comunidades expuestas; esos riesgos se incrementan si las tecnologías son deficientes en el control de emisiones y las poblaciones receptoras muestran condiciones de vulnerabilidad como pobreza, escaso acceso a salud y la presencia de grupos susceptibles como niños, embarazadas y adultos mayores.

Los principales contaminantes emitidos a partir de los procesos de una CTE son material particulado (MP), dióxido de nitrógeno $\left(\mathrm{NO}_{2}\right)$, dióxido de azufre $\left(\mathrm{SO}_{2}\right)$, dióxido de carbono $\left(\mathrm{CO}_{2}\right)$ y metales pesados como el mercurio $(\mathrm{Hg})$; adicionalmente, las emisiones de $\mathrm{NO}_{2}$ y $\mathrm{SO}_{2}$ son precursores en la atmósfera de ácido nítrico y sulfúrico ${ }^{1}$.

Los contaminantes del carbón afectan los principales órganos corporales y contribuyen en cuatro de las cinco principales causas de mortalidad en Estados Unidos: enfermedades cardíacas, cáncer, accidentes cerebro-vasculares y enfermedades crónicas respiratorias. En cada paso del ciclo de vida del carbón -extracción, transporte, lavado, combustión y desecho de residuos postcombustión- se producen emisiones que pueden influir en la salud de las personas. Se ha reportado que interfiere con el desarrollo pulmonar, incrementa el riesgo de infarto y afecta la capacidad intelectual, entre otros efectos ${ }^{2}$.

En Chile existe poca evidencia de daños en salud poblacional a pesar de que aún existen muchas insta- laciones de CTE a carbón. La evidencia internacional muestra que los riesgos asociados a este tipo de fuentes son altos y con impactos a lo largo de todo el ciclo vital especialmente en niños menores de 15 años y embarazadas.

El objetivo de este estudio fue realizar una revisión de la literatura científica nacional e internacional que reporte efectos en salud en población menor de 18 años, asociados a la exposición a emisiones provenientes de CTE a carbón.

\section{Método}

La búsqueda de publicaciones se realizó utilizando la base de datos bibliográficos electrónicos PubMed. Para la estrategia de búsqueda se consideró: exposición a contaminantes provenientes de CTE a carbón, efectos en salud de población general y menores de 18 años.

\section{Criterios de inclusión y exclusión de los estudios}

Se incluyeron todo tipo de estudios epidemiológicos que contemplaran la medición de exposición de contaminantes generados por CTE a carbón y efectos en salud en población general y en menores de 18 años de edad, sin límite de fecha de publicación y publicados en idioma inglés, español o portugués.

Fueron excluidos los estudios relacionados con exposiciones por combustión de carbón doméstica u otra fuente de emisión y publicaciones sin acceso a texto completo desde la Biblioteca UC (www.sibuc.cl).

\section{Análisis crítico de los estudios}

Los títulos identificados en la búsqueda fueron revisados de forma independiente por 2 de las autoras, 
quienes seleccionaron citas relacionadas al objetivo de la revisión, descartando aquellas no asociadas al objetivo del estudio. Posteriormente, todas las autoras de forma independiente realizaron la revisión de los resúmenes para verificar su pertinencia con el objetivo y el cumplimiento de los criterios de inclusión y exclusión. Cada autora completó esta evaluación en una planilla ad hoc y luego éstas fueron comparadas de forma cruzada para determinar los resúmenes elegibles para la revisión; cualquier discrepancia se resolvió mediante consenso. Después de esta primera etapa de selección, se accedió al texto completo de todos los artículos seleccionados para confirmar su elegibilidad y extraer la información pertinente.

Se elaboró una segunda planilla para realizar la extracción y síntesis de los resultados que incluyó información sobre la identificación de la publicación, método, población estudiada, definición de exposición y desenlace, principales resultados y limitaciones del estudio.

\section{Resultados}

La figura 1 muestra el flujo de las publicaciones en la búsqueda y selección de artículos que permitieron responder al objetivo de esta revisión. Se destaca el es-

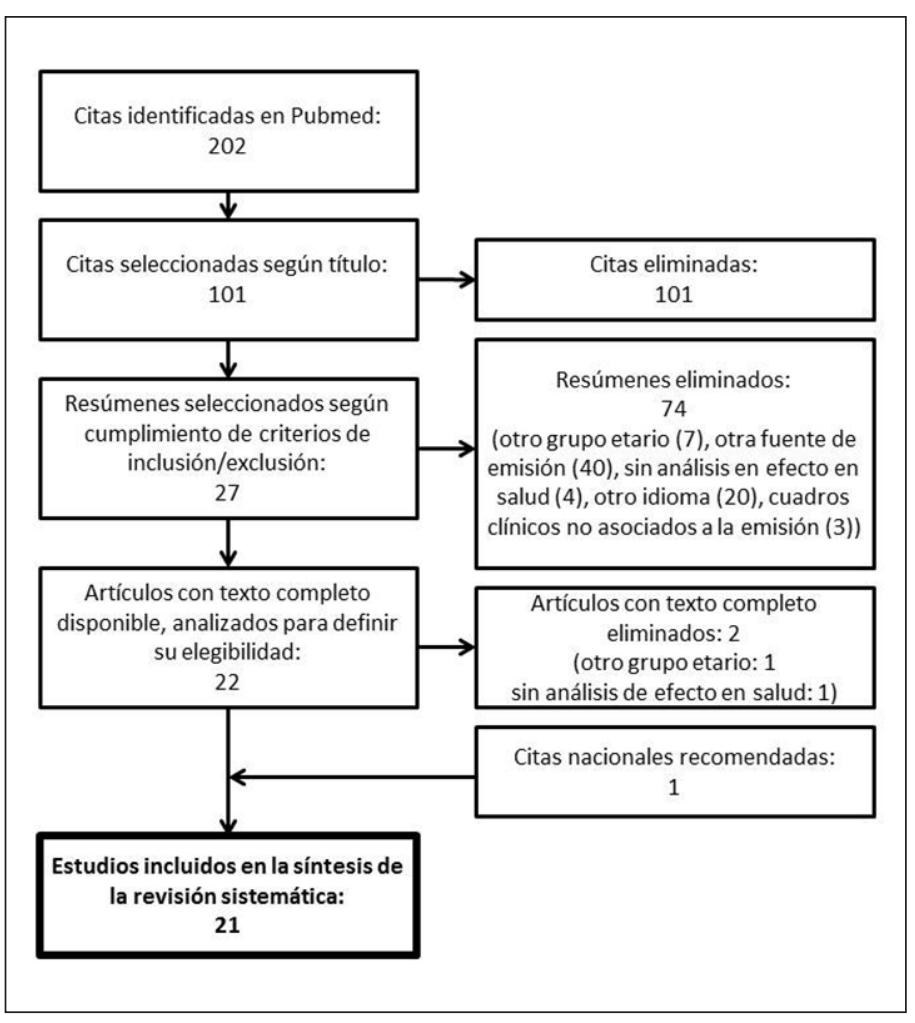

Figura 1. Flujo de la búsqueda y artículos incluidos en la revisión. caso número de publicaciones científicas encontradas sobre esta temática. Por otro lado, una fracción importante de artículos fue excluido por no abordar el objetivo del estudio (101/202, 50\%) o no cumplir los criterios de inclusión o exclusión (74/202, 36,6\%).

La tabla 1 muestra los artículos incluidos en la revisión con sus principales características y resultados. Se identificaron 4 aspectos asociados al daño en salud infantil: biomarcadores de exposición o efecto, efectos perinatales, desarrollo neurocognitivo y salud respiratoria.

Se presentan a continuación los principales hallazgos de la literatura.

\section{Biomarcadores}

Los 5 estudios analizados fueron consistentes en el reporte de una mayor presencia de biomarcadores de exposición y de efecto temprano en las zonas expuestas cercanas a CTE con combustión a carbón, en comparación con zonas no expuestas.

Estudios de cohorte en los que se evaluaron mujeres no fumadoras con residencia dentro de $2,5 \mathrm{~km}$ de una CTE a carbón y sus recién nacidos, evaluados en el año 2002 y 2005, compararon biomarcadores moleculares de exposición y de efecto preclínico en sangre de cordón umbilical en relación a varios desenlaces del recién nacido (peso, talla, circunferencia craneana (CC), neurodesarrollo y Coeficiente Intelectual (CI)), antes y después del cierre de operaciones de una CTE. La cohorte de niños nacidos 2 años después del cierre de la planta mostró: aumento en la CC, reducción en el nivel de aductos de ADN y en los niveles sanguíneos y ambientales de Hidrocarburos Aromáticos Policíclicos (HAP), concentraciones más altas de proteína BDNF madura (mBDNF) y puntuaciones más altas del Coeficiente de Desarrollo $(\mathrm{CD})^{3-7}$.

Estudios transversales evaluaron los niveles urinarios de metales pesados y 1-hidroxipireno (1-OHP) como biomarcadores de exposición, y 8-OHdG, HNEMA, 8-isoPGF2 $\alpha$ y 8 -NO2Gua como biomarcadores de efecto temprano en niños de 9 a 15 años residentes en la cercanía de refinerías de petróleo y CTE a carbón. Se reportó que aquellos con residencia más cercana, tuvieron concentraciones ambientales elevadas de vanadio e HAP, altos niveles urinarios de 1-OHP y metales pesados y concentraciones más altas de los biomarcadores de estrés oxidativo en orina, en comparación con los sujetos de baja exposición ${ }^{8}$.

Otro estudio realizado en China con niños entre 1 a 13 años, reportó que niños que viven en comunidades de alta exposición tuvieron concentraciones urinarias de 1-OHP más altas que aquellos de comunidades de baja exposición, resultados consistentes al ajustar por edad, sexo, humo de tabaco ambiental, dieta y exposición al tráfico?. 


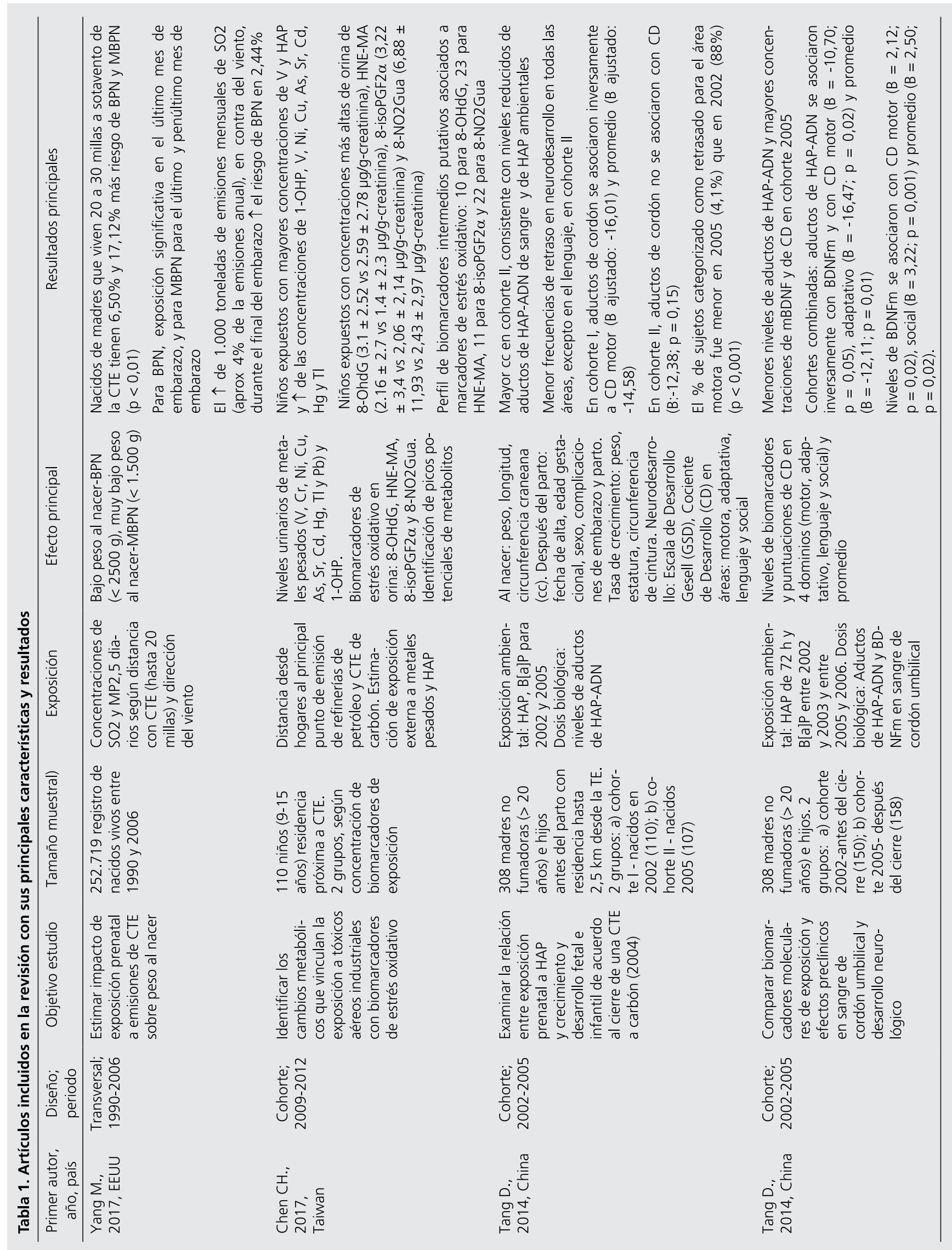




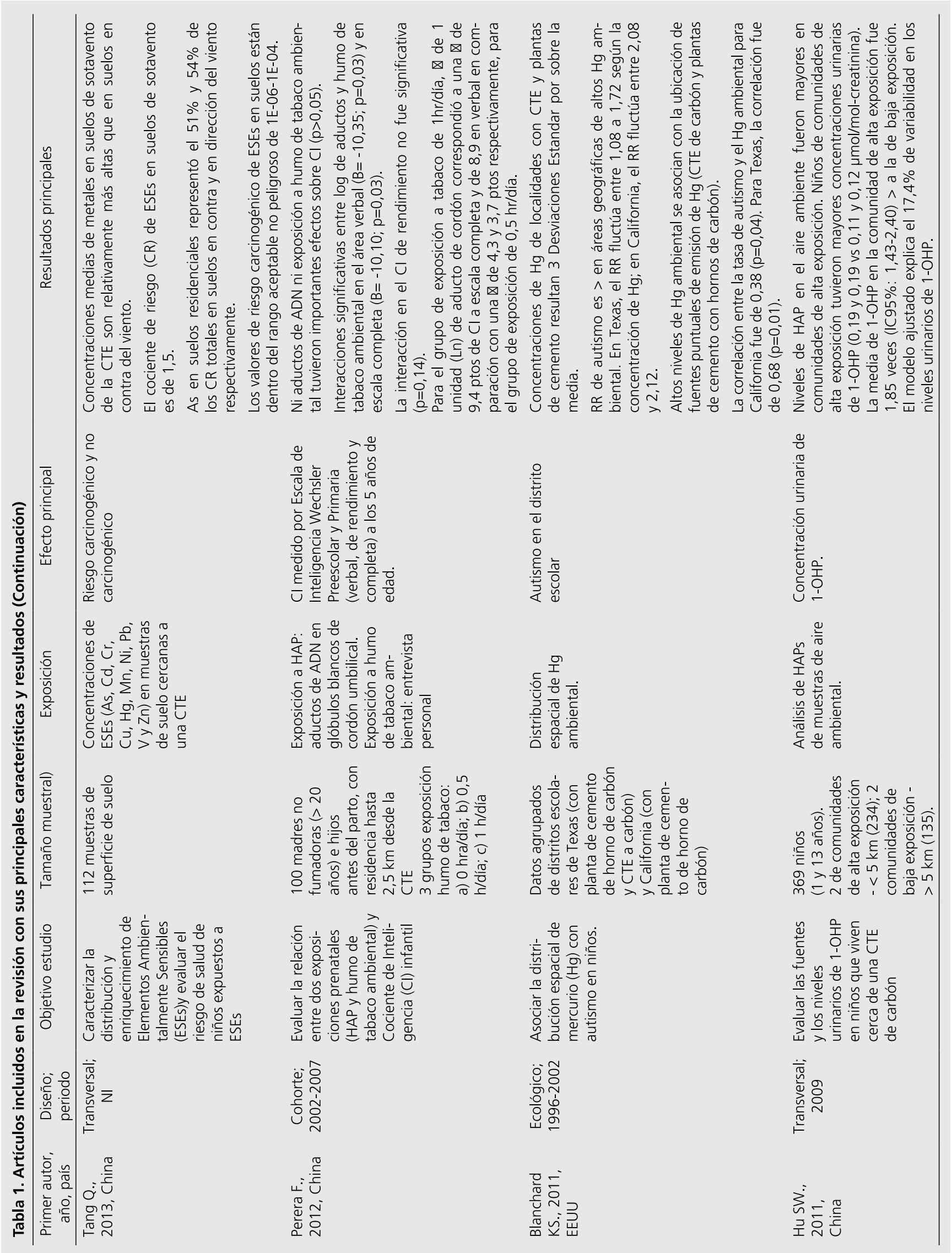




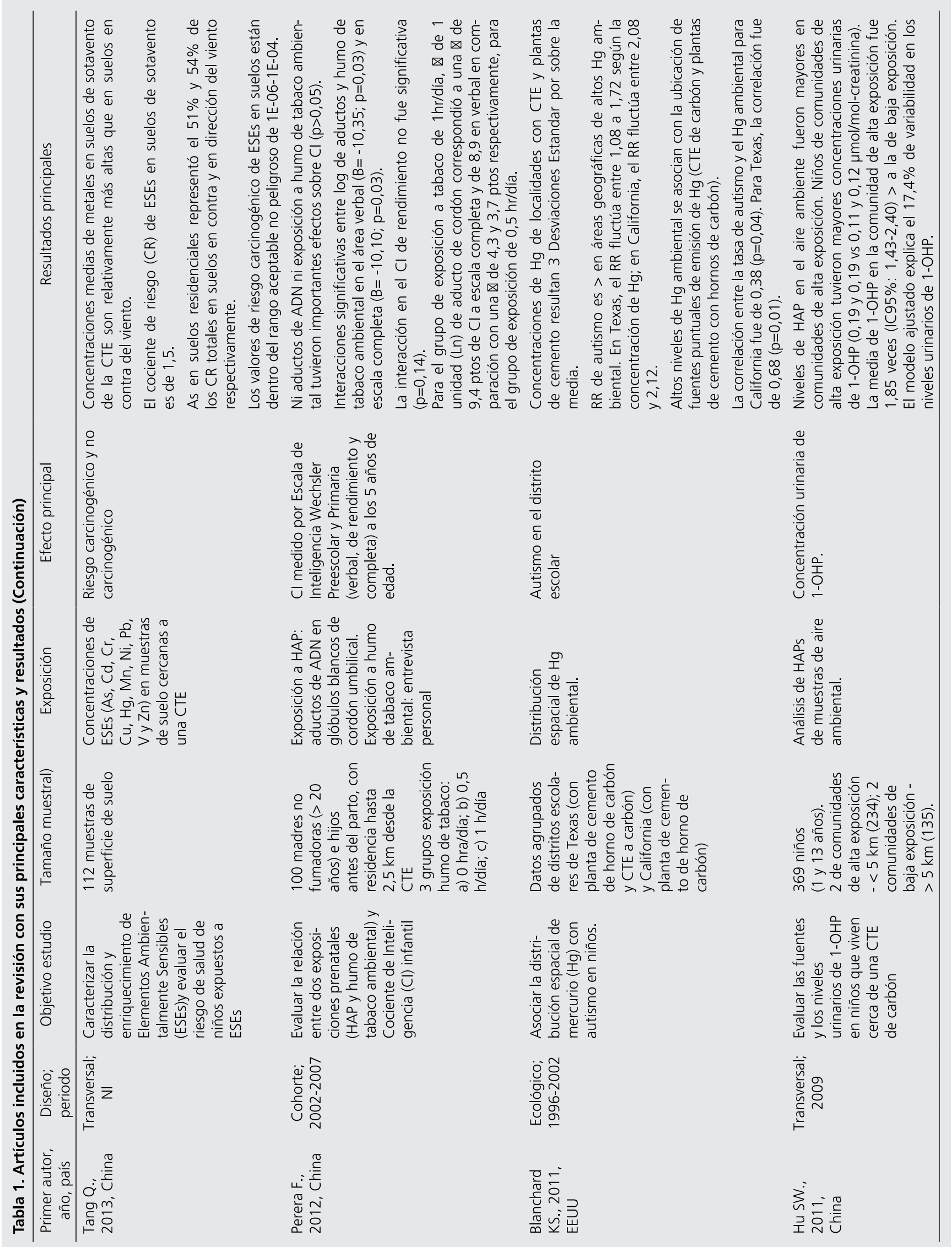




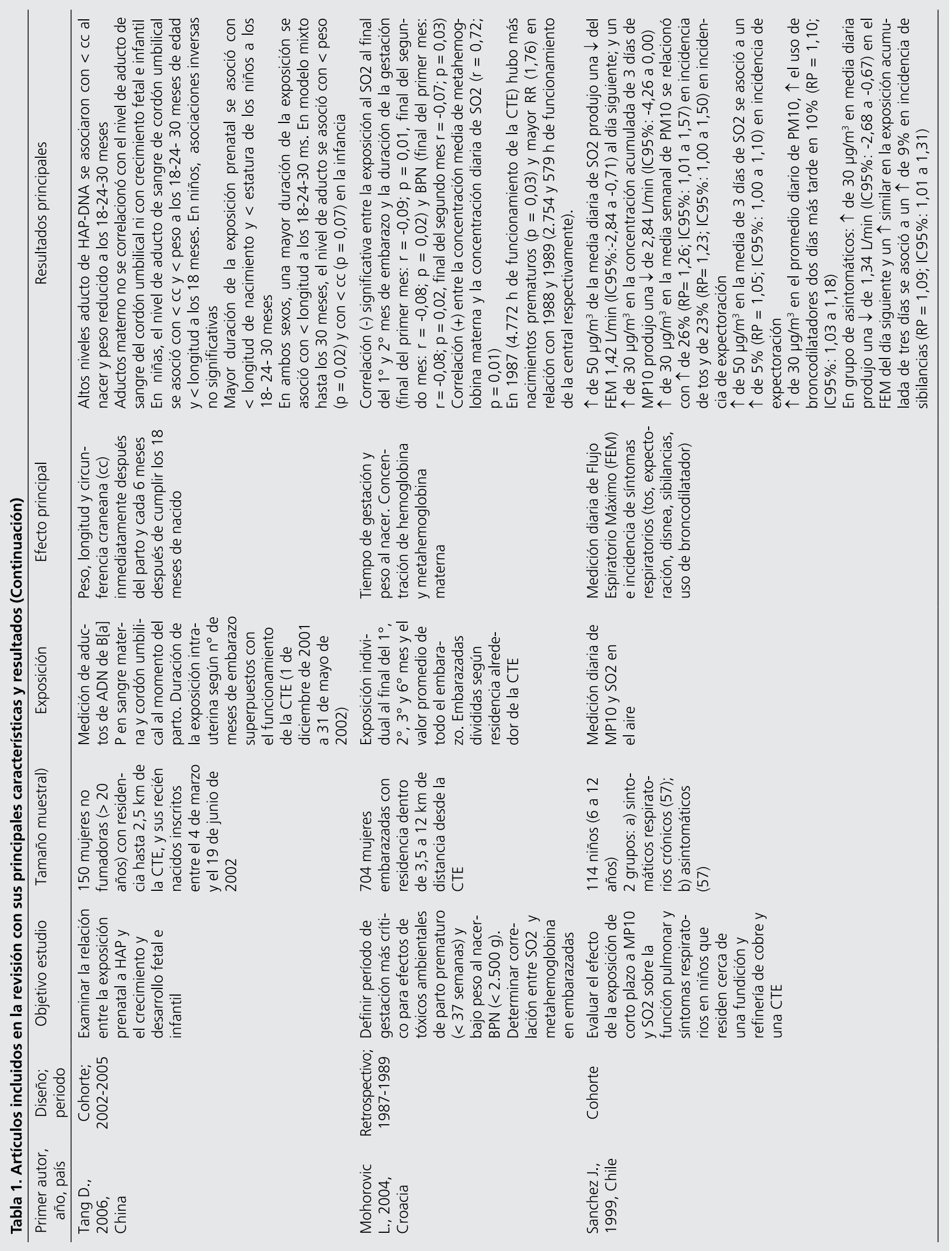




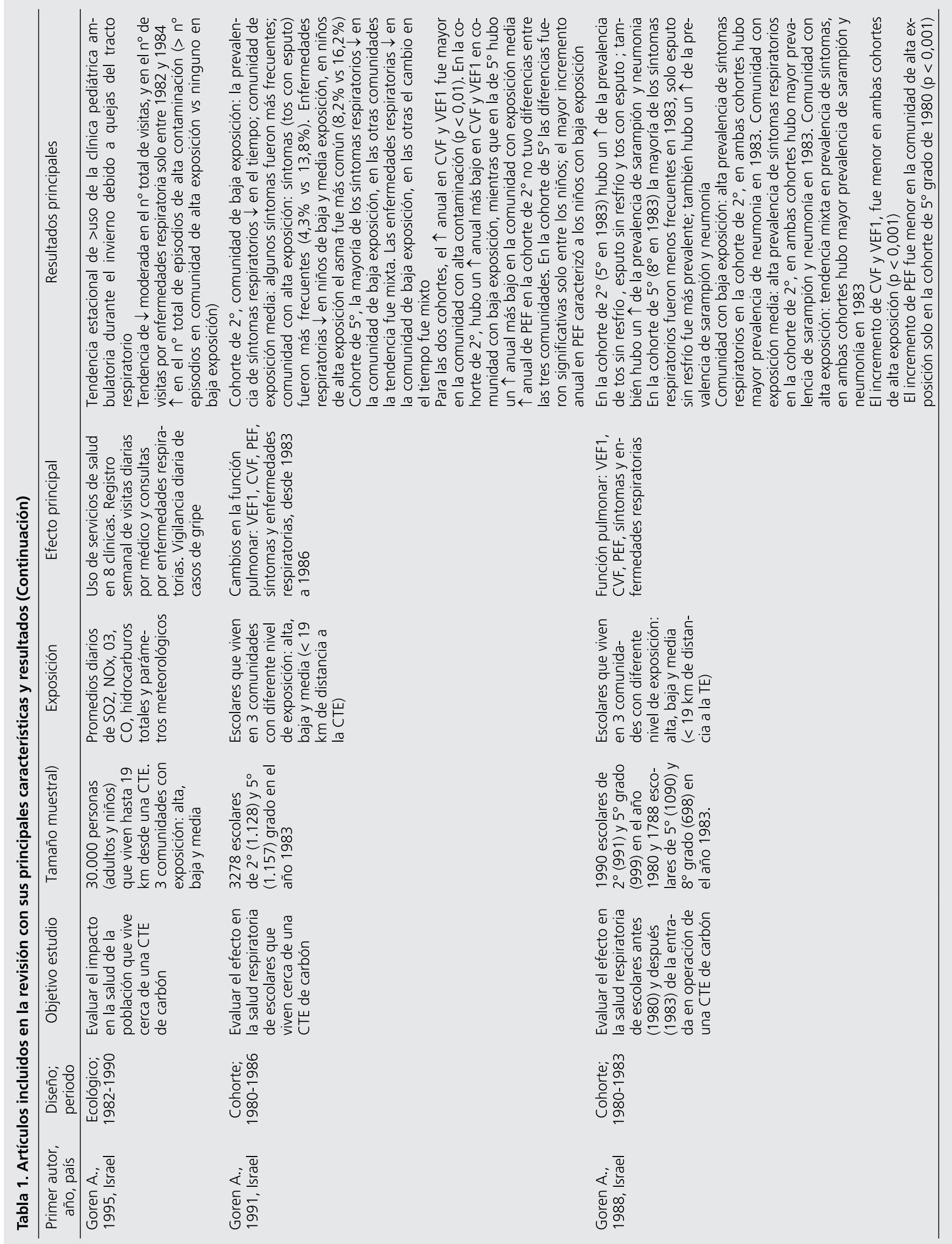




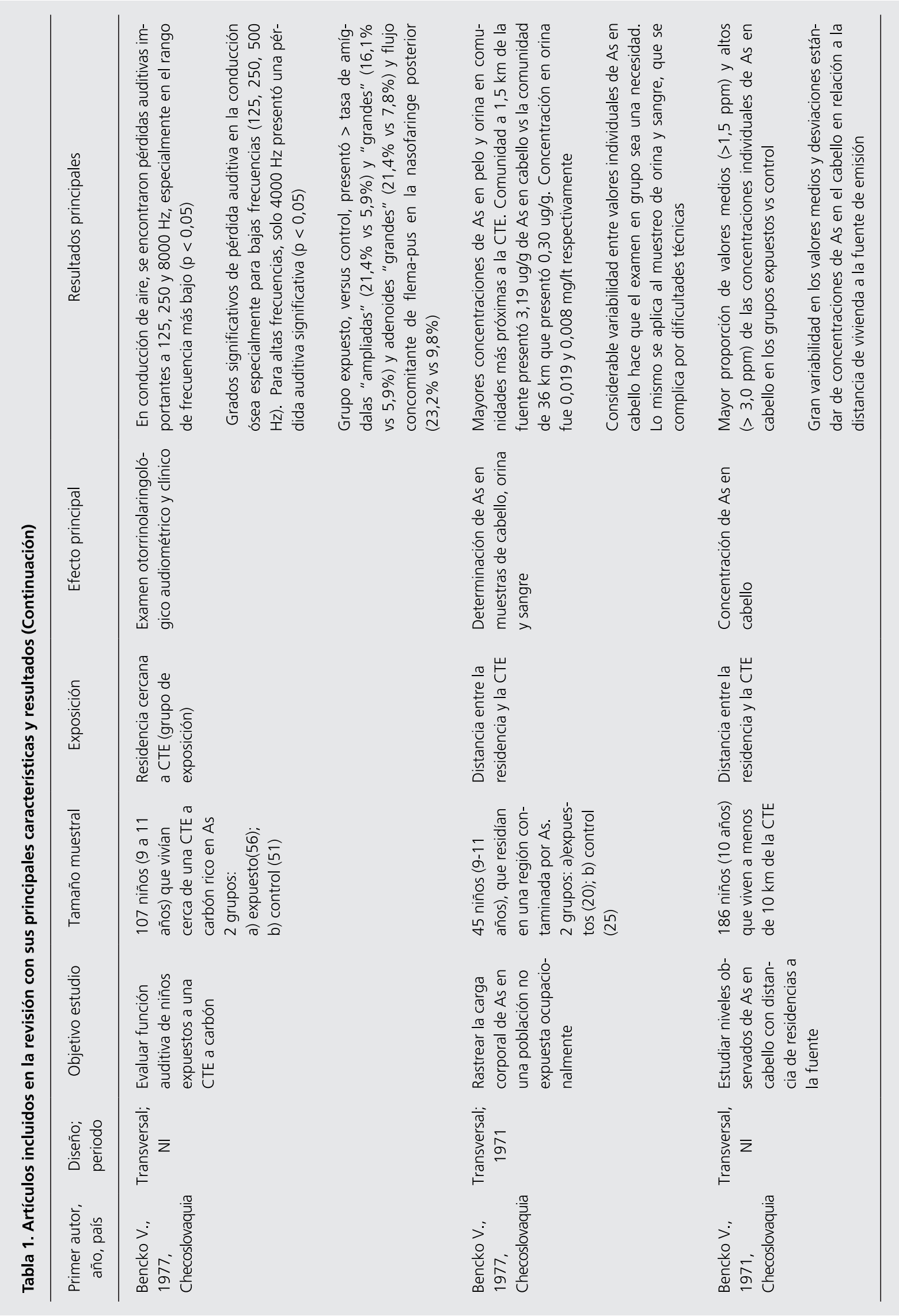




\section{Alteraciones perinatales}

Los 4 estudios analizados reportaron que la exposición en el embarazo, evaluada a través de la distancia desde la residencia a las emisiones de CTE a carbón, se asoció a recién nacidos $(\mathrm{RN})$ con bajo y muy bajo peso (BP y MBP, respectivamente), menor talla al nacimiento, gestación más corta y menor diámetro de CC. En el estudio realizado por Tangs y cols. se reporta que la CC aumentó en niños nacidos después del cierre de la $\mathrm{CTE}^{4,10-12}$.

Yang (2017) estudió el impacto de la exposición prenatal a las emisiones de CTE a carbón sobre el peso al nacer en Estados Unidos entre 1990 y 2006 (252.719 $\mathrm{RN}$ ), reportando que los nacidos de madres que viven a una distancia de $5 \mathrm{~km}$ aproximadamente de la CTE tienen un mayor riesgo de $\mathrm{BP}(6,5 \%)$ y $\operatorname{MBP}(17,1 \%)^{10}$.

Mohorovic (2004) estudió embarazadas categorizadas según la distancia de su residencia a una CTE a carbón en Croacia, definiendo zonas concéntricas alrededor de ésta (entre 3,5-12 km). Sus hallazgos evidenciaron que una exposición mayor y más prolongada a emisiones de $\mathrm{SO}_{2}$ durante los 2 primeros meses de embarazo resultó en una gestación significativamente más corta y en menor peso al nacer ${ }^{11}$.

En China, fueron desarrollados dos estudios prospectivos que analizaron efectos perinatales de la exposición a la emisión de una CTE ubicada a menos de 5 $\mathrm{km}$ de distancia de la vivienda de mujeres durante el embarazo. El primero de éstos reportó que los hijos de madres que vivían cerca de la CTE se caracterizaban por menor CC y menor peso en los meses posteriores al nacimiento. Una exposición más prolongada en el embarazo se asoció con menor talla al nacer y a los 18, 24 y 30 meses de edad $(\mathrm{p}<0,001)^{12}$. Posteriormente, los mismos autores, compararon resultados entre esta cohorte (nacidos durante el funcionamiento de la central) y una nueva (nacidos después del cierre de la central), entre los resultados se destacó un mayor CC para aquellos niños nacidos posterior al cierre de CTE de carbón $(\mathrm{p}<0,001)^{4}$.

\section{Desarrollo neurocognitivo}

Los artículos incluidos en la revisión apuntan a una disminución del CD y CI en los niños expuestos a emisiones de CTE a carbón, comparados con niños no expuestos.

Seis estudios evaluaron los efectos de la exposición a contaminantes emitidos por CTE a carbón sobre el desarrollo neurocognitivo: 5 de ellos se basaron en registros de una cohorte de niños cuyas madres desde el inicio del embarazo residían cerca de CTE de Tongliang, China. En 4 de estos estudios el seguimiento consideró el periodo desde el embarazo hasta 2 años de edad, y solo un estudio hasta los 5 años ${ }^{3-4,6,7,13,14}$.

Tras el seguimiento de 2 años a los nacidos el año
2002, con la central en pleno funcionamiento, se evaluó la Pauta de Desarrollo Gesell para determinar el $\mathrm{CD}$ en 4 dominios (motor, adaptativo, lenguaje y social). Se observó una disminución en CD motor y en el promedio de todos los dominios asociados a una mayor concentración de biomarcadores para HAP en sangre del cordón umbilical, mientras, el CD social se asoció negativamente con las concentraciones de plomo en sangre del cordón umbilical, ambas sustancias detectadas en las emisiones de la CTE a carbón? ${ }^{7}$. Un estudio posterior comparó los resultados de CD de la misma cohorte con otros nacidos en 2005, tras el cierre de CTE. En dicho estudio se encontró una reducción significativa de biomarcadores para HAP en sangre de cordón umbilical de niños de la cohorte de 2005, encontrándose una asociación inversa entre la concentración de biomarcadores y el CD en el dominio motor y en el promedio para la cohorte más vieja, relación no observada en la cohorte más nueva ${ }^{13}$.

A los 5 años de edad de los niños de la cohorte se evaluó el CI mediante la Escala de Inteligencia Wechsler preescolar y primaria, en ella se consideraron 3 escalas: verbal, de rendimiento y completa. Ni los aductos de ADN ni la exposición al humo de tabaco ambiental tuvieron importantes efectos principales sobre el CI. Sin embargo, hubo interacciones significativas entre aductos y humo de tabaco ambiental en la escala completa $(\mathrm{p}=0,025)$ y en el área verbal $(\mathrm{p}=0,029)$, lo que indica que los efectos adversos de la exposición prenatal a HAP aumentaron a medida que aumentaba la exposición al humo de tabaco ambiental ${ }^{6}$.

En la cohorte de niños no expuestos a las emisiones de la central (nacidos el 2005) se reportaron concentraciones más altas de la proteína mBDNF y mayores puntuaciones de CD a los 2 años de edad ${ }^{3}$. Otro estudio destacó que las frecuencias de retraso en el neurodesarrollo en casi todas las áreas (excepto lenguaje), se redujeron en la cohorte 2005 en comparación con la del $2002^{4}$.

Un estudio ecológico realizado en Estados Unidos, analizó la distribución espacial de autismo en relación a la contaminación ambiental por mercurio. Los autores reportaron mayor riesgo de autismo para zonas con fuentes de emisión de mercurio (CTE carbón y plantas de cemento con hornos de carbón). La correlación entre la tasa de autismo y los niveles de mercurio en el aire ambiente para California fue de 0,38 $(\mathrm{p}=0,04)$, mientras que para Texas fue de $0,68(\mathrm{p}=0,01)$. Debe considerarse este resultado con cautela, dado las limitaciones propias de los estudios ecológicos ${ }^{14}$.

\section{Salud respiratoria}

Del total de estudios incluidos, se reportan cambios en la función pulmonar, síntomas respiratorios y consultas ambulatorias por causas respiratorias. 
Los estudios que evaluaron los efectos en la función pulmonar utilizaron principalmente el Volumen Espiratorio Forzado en el primer segundo (VEF1), la Capacidad Vital Forzada (CVF) y el Flujo Espiratorio Máximo (PEF), y se enfocaron en efectos de largo plazo. El estudio de Dubnov J. et al. (2007), enfocado en evaluar el efecto de la exposición a largo plazo de la contaminación proveniente de CTE a carbón sobre el desarrollo de la función pulmonar en una cohorte de escolares de $2^{\circ}, 5^{\circ}$ y $8^{\circ}$ grado según su residencia seguidos entre 1996 y 1999, reportó que la concentración de $\mathrm{NO}_{2}$ tuvo un efecto negativo significativo tanto en $\triangle V E F 1$ como en $\triangle \mathrm{CVF}$, lo que significa que a mayor exposición existe un menor crecimiento de la función pulmonar. Por otro lado, el efecto de la interacción $\mathrm{NO}_{2}-\mathrm{SO} 2$ en $\triangle \mathrm{VEF} 1$ fue negativo y más fuerte que la variable $\mathrm{NO}_{2}$ por sí sola. Además, al excluir la variable contaminación se redujo el poder explicativo de los modelos en $6 \%$ y $10 \%$, lo que confirma que la contaminación explica las variables de efecto estudiadas ${ }^{15}$.

El estudio de Yogev-Baggio et al. (2010) corresponde a la misma cohorte anteriormente descrita, pero en este reporte se muestra un análisis según estado de salud de los escolares (sanos, sintomáticos respiratorios y asmáticos). En cuanto al estado de salud entre 1996 y 1999 se destaca que el porcentaje de niños sanos disminuyó y el de sintomáticos aumentó en todas las áreas de exposición estudiadas (siendo de mayor magnitud en la de alta exposición). Se observaron diferencias significativas en el $\triangle \mathrm{VEF} 1$ de los 3 grupos según área de contaminación (aunque a mayor contaminación, mayor disminución $\triangle \mathrm{VEF} 1$ ) y el efecto de la interacción $\mathrm{NO}_{2}-\mathrm{SO}_{2}$ en $\triangle \mathrm{VEF} 1$ fue negativa y significativa para todos los niños, y grupo de sanos y sintomáticos $(\mathrm{p}<0,01)$, esto implica que los niveles crecientes de contaminación tienen un efecto negativo significativo en el crecimiento de la función pulmonar ${ }^{16}$.

Goren et al. (1988 y 1991) evaluaron la función pulmonar en escolares que viven cerca de CTE a carbón donde las primeras mediciones se realizaron antes de que la central comenzara a funcionar (1980) y 3 y 6 años después (1983 y 1986). Los escolares se clasificaron en comunidades con alta, media y baja exposición. En el primer reporte se encontró que el incremento de CVF y VEF1 fue menor en ambas cohortes en la comunidad de alta exposición y que el incremento de PEF fue menor en la comunidad de alta exposición solo en la cohorte de $5^{\circ}$ grado ( $8^{\circ}$ en 1983). En el segundo reporte, se encontró un aumento anual en CVF y VEF1 mayor en la comunidad con alta contaminación. La cohorte de $2^{\circ}$ en 1983 , tuvo un aumento anual menor en CVF y VEF1 en la comunidad con baja exposición, mientras que la cohorte $5^{\circ}$ en 1983 tuvo un aumento anual menor en la comunidad con exposición media, por otro lado el aumento anual de PEF en la cohorte de $2^{\circ}$ no tuvo diferencias entre las tres comunidades ${ }^{17,18}$.

Al evaluar sólo síntomas, el primer reporte (1988) mostró que la cohorte de $2^{\circ}$ ( $5^{\circ}$ en 1983) tuvo un aumento significativo de la prevalencia de tos sin resfrío. En la cohorte de $5^{\circ}$ ( $8^{\circ}$ en 1983 ) la mayoría de los síntomas respiratorios fueron menos frecuentes en 1983. Con respecto a las comunidades según exposición, el grupo con baja exposición tuvo alta prevalencia de síntomas respiratorios en la cohorte más joven y en ambas cohortes hubo mayor prevalencia de neumonía en 1983, el grupo con exposición media tuvo alta prevalencia de síntomas respiratorios en la cohorte más joven y en ambas cohortes hubo mayor prevalencia de sarampión y neumonía en 1983, y el grupo con alta exposición tuvo tendencia mixta en la prevalencia. En el segundo reporte (1991), en el grupo de baja exposición la prevalencia de la mayoría de los síntomas respiratorios se redujo cuando los niños crecieron, en el grupo de exposición media algunos síntomas fueron más frecuentes, y en el grupo con alta exposición los síntomas fueron significativamente más frecuentes ${ }^{17,18}$.

Un estudio ecológico realizado en Israel estableció aumentos en las consultas pediátricas respiratorias en la población expuesta a una CTE, asociados a aumentos de la contaminación del aire ${ }^{19}$.

\section{Otros desenlaces en salud}

En población infantil se han reportado otros daños, entre ellos, pérdidas auditivas en niños que vivían próximos a una CTE a carbón al compararlos con niños no expuestos; los mismos autores reportaron mayores niveles de arsénico en muestras biológicas en los niños expuestos; destacan la variabilidad de estos biomarcadores ${ }^{20-22}$.

\section{Conclusiones}

La revisión de la literatura para dar respuesta al objetivo planteado, incluyó la revisión de 21 artículos de texto completo que fueron pertinentes al objetivo y cumplieron los criterios de inclusión y exclusión.

Los estudios incluidos que evaluaron biomarcadores fueron consistentes en el reporte de un aumento en el nivel de biomarcadores de exposición y de efecto en salud en zonas expuestas cercanas a instalaciones que funcionan con combustión a carbón en comparación con zonas no expuestas.

En relación a las alteraciones perinatales, los estudios reportaron que la exposición a emisiones de CTE a carbón en el embarazo se asoció a niños con BP y $\mathrm{MBP}$ al nacer, menor talla, menor diámetro de CC y prematuridad. Por otro lado, el diámetro de CC aumentó en los niños nacidos después del cierre de CTE. 
Con respecto al desarrollo cognitivo se encontró tanto un CD y CI inferior en los niños expuestos a emisiones de CTE a carbón comparados con no expuestos, y un aumento del CD en una cohorte de niños que creció cuando la central se había cerrado. Por otro lado, vivir en zonas con fuentes de emisión de mercurio (CTE y plantas de cemento que funcionan con carbón) se asoció con un mayor riesgo de autismo.

En salud respiratoria, los artículos fueron consistentes en reportar un menor desarrollo de la función pulmonar a largo plazo en niños residentes en zonas expuestas a fuentes de combustión de carbón comparados con grupos de niños no expuestos. Otros estudios reportaron una mayor frecuencia de síntomas respiratorios (tos, sibilancias y dificultad para respirar), mayor prevalencia de sarampión y neumonía, y más diagnósticos de asma en los niños que viven en las comunidades expuestas comparados con menor o sin exposición.

Existe una fuerte preocupación de la comunidad científica con respecto a la exposición a CTE a carbón y efectos en la salud infantil. Esta revisión encontró diversos efectos en la salud infantil relacionados con la presencia de biomarcadores de exposición y efecto, efectos perinatales, neuroconductuales y respiratorios principalmente. Es de suma urgencia el desarrollo de estudios enfocados en este tema de alta sensibilidad y de alto impacto en salud pública, destacándose que hasta ahora sólo existe limitada evidencia generada por Ruiz-Rudolph et al. (2016) quienes demostraron que la presencia de grandes instalaciones (entre ellas CTE a carbón y petróleo, fábricas de pastas de celulosa, mineras y fundiciones de cobre) aumentaban las tasas de mortalidad y morbilidad, en un rango de 20 a $100 \%$ en aquellas comunas que tenían megafuentes con respecto a las comunas sin este tipo de exposición ${ }^{23}$.

Es perentorio abrir en Chile el debate sobre los riesgos controlables a los que se enfrenta la población infantil a consecuencia de plantas generadoras de energía instaladas en Chile.

\section{Agradecimientos}

Las autoras expresan su agradecimiento a la Sociedad Chilena de Pediatría y a Chile Sustentable por facilitar las condiciones necesarias para realizar esta investigación y por proveer información ambiental de interés.

\section{Conflicto de interés}

Las autoras declaran no tener conflicto de interés.

\section{Referencias}

1. Health and Environment Allience (HEAL). Boosting health by improving air quality in the Balkans. Published Diciembre 2017 [revisado el 19 de enero de 2018]. Disponible en https://www. env-health.org/IMG/pdf/13.12.2017_-_ boosting_health_by_improving_air_ quality_in_the_balkans_ied_briefing.pdf

2. Gohlke, J. M., et al. Estimating the global public health implications of electricity and coal consumption. Environ Health Perspect. 2011;119(6):821-6.

3. Tang D, Lee J, Muirhead L, et al. Molecular and neurodevelopmental benefits to children of closure of a coal burning power plant in China. PLoS ONE. 2014;9(3):e91966.

4. Tang D, Li TY, Chow JC, et al. Air pollution effects on fetal and child development: a cohort comparison in China. Environ Pollut. 2014;185:90-6.

5. Tang Q, Liu G, Zhou C, Zhang H, Sun R. Distribution of environmentally sensitive elements in residential soils near a coal-fired power plant: potential risks to ecology and children's health. Chemosphere. 2013;93(10):2473-9.

6. Perera F, Li TY, Lin C, Tang D. Effects of prenatal polycyclic aromatic hydrocarbon exposure and environmental tobacco smoke on child IQ in a Chinese cohort. Environ Res. 2012;114:40-6.

7. Tang D, Li T, Liu JJ, et al. Effects of prenatal exposure to coal-burning pollutants on children's development in China. Environ Health Perspect. 2008;116(5):674-9.

8. Chen C-HS, Yuan T-H, Shie R-H, Wu $\mathrm{K}-\mathrm{Y}$, Chan C-C. Linking sources to early effects by profiling urine metabolome of residents living near oil refineries and coal-fired power plants. Environ Int. 2017;102:87-96.

9. Hu S-W, Chan Y-J, Hsu H-T, et al. Urinary levels of 1-hydroxypyrene in children residing near a coal-fired power plant. Environ Res. 2011;111(8):1185-91.

10. Yang M, Bhatta RA, Chou S-Y, Hsieh C-I. The Impact of Prenatal Exposure to Power Plant Emissions on Birth Weight: Evidence from a Pennsylvania Power Plant Located Upwind of New Jersey. J Policy Anal Manage. 2017;36(3):557-83.

11. Mohorovic L. First two months of pregnancy-critical time for preterm delivery and low birthweight caused by adverse effects of coal combustion toxics. Early Hum Dev. 2004;80(2):115-23.

12. Tang D, Li T, Liu JJ, Chen Y, Qu L, Perera F. PAH-DNA adducts in cord blood and fetal and child development in a Chinese cohort. Environ Health Perspect. 2006;114(8):1297-300.

13. Perera F, Li T, Zhou Z, et al. Benefits of reducing prenatal exposure to coal-burning pollutants to children's neurodevelopment in China. Environ Health Perspect. 2008;116(10):1396-400.

14. Blanchard KS, Palmer RF, Stein Z. The value of ecologic studies: mercury concentration in ambient air and the risk of autism. Rev Environ Health. 2011;26(2):111-8.

15. Dubnov J, Barchana M, Rishpon S, et al. Estimating the effect of air pollution from a coal-fired power station on the development of children's pulmonary function. Environ Res. 2007;103(1):87-98.

16. Yogev-Baggio T, Bibi H, Dubnov J, Or-Hen K, Carel R, Portnov BA. Who is affected more by air pollution-sick or healthy? Some evidence from a health survey of schoolchildren living in the vicinity of a coal-fired power plant in Northern Israel. Health Place. 2010;16(2):399-408.

17. Goren AI, Helman S, Goldsmith JR. Longitudinal study of respiratory conditions among schoolchildren in Israel: interim report of an epidemiological monitoring program 
in the vicinity of a new coal-fired power plant. Arch Environ Health. 1988;43(2):190-4.

18. Goren AI, Goldsmith JR, Hellmann S, Brenner S. Follow-up of schoolchildren in the vicinity of a coal-fired power plant in Israel. Environ Health Perspect. 1991;94:101-5.

19. Goren AI, Hellmann S, Glaser ED. Use of outpatient clinics as a health indicator for communities around a coal-fired power plant. Environ Health Perspect. 1995;103(12):1110-5

20. Bencko V, Dobišová A, Máčaj M. Arsenic in the hair of a non-occupationally exposed population. Atmospheric Environment.1971;5(4):275-9.

21. Bencko V, Symon K, Chládek V, Pihrt J. Health aspects of burning coal with a high arsenic content: II. Hearing changes in exposed children. Environmental Research. 1977;13(3):386-95.
22. Bencko V, Symon K. Health aspects of burning coal with a high arsenic content: I. Arsenic in hair, urine, and blood in children residing in a polluted area. Environmental Research. 1977;13(3):37885.

23. Ruiz-Rudolph P, Arias N, Pardo S, et al. Impact of large industrial emission sources on mortality and morbidity in Chile: A small-areas study. Environ Int. 2016;92-93:130-8. 